\title{
$\&$ Evolving utility of bibliometric indices in pediatric neurosurgery
}

\author{
Jeffrey P. Blount, MD \\ Division of Pediatric Neurosurgery, University of Alabama at Birmingham, Alabama
}

I $\mathrm{N}$ their paper "The 50 most cited publications in endoscopic third ventriculostomy: a bibliometric analysis," Zagzoog and colleagues present a bibliometric study on endoscopic third ventriculostomy (ETV) and detail the 50 most cited references in the field. They identified 1200 articles about ETV published between 1966 and 2016 and reduced their number to 494 after manually discarding those papers not directly related to the procedure. The 50 most highly cited articles were identified, as were the top 20 articles with the highest citation rate per year. For each article, the total number of citations, citations per year, journal of publication, and authors' country of record were recorded. They stratified the articles into 4 different categories: 1) clinical studies, 2) reviews, 3) radiological articles, and 4) technical papers and then further sub-stratified the articles by patient age to indicate pediatric, mixed pediatric and adult, and adult series within the categories above. Results were tallied, and basic descriptive statistics for the two cohorts (top 50 and most frequently cited 20) were calculated. The mean number of total citations for the top 50 articles was 141 (mean 9 citations/yr for top 50 ), but only one article had more than 400 citations, thus meeting the criteria for a classic article. Seven of the 50 most cited articles failed to make the list of most frequently referenced, and all were older studies. Clinical articles were the most heavily cited, and the majority of these were purely pediatric. The current study provides a valuable addition to the expanding literature on bibliometric analyses in neurosurgery. Future work should expand such analyses specifically in terms of sample size or quality of evidence produced.

In an era in which there is explosive growth in the sheer number of manuscripts published, metrics for assessing impact are important. The biggest shortcoming of citation tallies as the preferred bibliometric is its assumption that the number of citations reflects the overall contribution that the paper makes. This approach ignores issues of study design and the quality of the evidence that arises from a paper. Indeed, in many fields, the classic references have a study design that would struggle to reach the level for publication today. They have attained classic study status because of the creativity or novelty of the approach or technique described and the early timing of the publication. Thus, as the authors allude, many older papers that appeared early in the learning curve of a particular concept or approach are repeatedly cited in subsequent papers and thus gain a high citation index but remain of modest design and generate modest-quality evidence. This has the unfortunate outcome of perpetuating initial thoughts and entrenching concepts that are supported by studies of modest-quality evidence. Only the incorporation of standards of quality of evidence gets away from this. The JNS Publishing Group has acknowledged this important issue and now indicates with a graphic the level of evidence that results from the data and analysis within a paper.

More sophisticated bibliometric indices are available and, appropriately, are increasingly used as the preferred metric to assess individual, departmental, and program academic activity. 4,5 The h-index, or Hirsch index, is the most commonly used bibliometric as it combines the citation index and productivity into a single value. ${ }^{1,3}$ It was originally used for the comparison of individuals but has been increasingly used to compare academic departments and programs. ${ }^{3-5}$ The h-index has been refined to the ih(5)-index to reflect academic productivity in discrete 5 -year intervals. ${ }^{5}$ The failure of the h-index to adequately control for variable effort and contributions among authors led to the development of the r-index (replicability index). An important and recent study utilized the ih(5)index to compare the productivity of each of the Accreditation Council for Pediatric Neurosurgical Fellowships (ACPNF)-approved fellowship programs in pediatric neurosurgery. ${ }^{3}$ Just as study design and quality of evidence have improved, one can anticipate that bibliometric pa- 
rameters will and should increasingly define the measure of academic productivity in pediatric neurosurgery in the near future. ${ }^{2,3}$

https://thejns.org/doi/abs/10.3171/2018.6.PEDS18118

\section{References}

1. Khan N, Thompson CJ, Choudhri AF, Boop FA, Klimo P Jr: Part I: The application of the h-index to groups of individuals and departments in academic neurosurgery. World Neurosurg 80:759-765, 765.e1-765.e3, 2013

2. Klimo P Jr, Michael LM II, Venable GT, Taylor DR: Bibliometrics. J Neurosurg 124:877-878, 2016 (Letter)

3. Klimo P Jr, Venable GT, Khan NR, Taylor DR, Shepherd BA, Thompson CJ, et al: Bibliometric evaluation of pediatric neurosurgery in North America. J Neurosurg Pediatr 14:695-703, 2014

4. Lee RP, Venable GT, Roberts ML, Parikh KA, Taylor DR, Khan NR, et al: Five-year institutional bibliometric profiles for 119 North American neurosurgical residency programs: an update. World Neurosurg 95:565-575, 2016

5. Lee RP, Xu R, Dave P, Ajmera S, Lillard JC, Wallace D, et al: Taking the next step in publication productivity analysis in pediatric neurosurgery. J Neurosurg Pediatr 21:655-665, 2018

6. Zagzoog N, Attar A, Reddy K: The 50 most cited publications in endoscopic third ventriculostomy: a bibliometric analysis. J Neurosurg Pediatr [epub ahead of print October 19, 2018. DOI: 10.3171/2018.2.PEDS17354]

\section{Disclosures}

The author reports no conflict of interest.

\section{Correspondence}

Jeffrey P. Blount: jeffrey.blount@childrensal.org.

INCLUDE WHEN CITING

Published online October 19, 2018; DOI: 10.3171/2018.6.PEDS18118.

\section{Response}

\author{
Nirmeen Zagzoog, MSc, MD, ${ }^{1}$ \\ Ahmed Attar, MBBS, ABPN, FRCPC, ${ }^{2}$ and \\ Kesh Reddy, MBBS, FRCSC, DABNS ${ }^{1}$
}

1Department of Surgery, Division of Neurosurgery; and 2Department of Medicine, Division of Neurology, Hamilton General Hospital, McMaster University, Hamilton, Ontario, Canada

Dr. Blount's editorial on our paper raises some excellent points and highlights some of the limitations of bibliometric methods in the evaluation of literature. When performing our bibliometric evaluation, we employed Harzing's Publish or Perish algorithmic software ${ }^{3}$ and the Google Scholar database to perform a citation analysis within our defined parameters-the traditional and currently the most commonly used method for estimating the impact of publications. An analysis of the highest rates of citationthe greatest number of citations in a single year-was also completed as a way of correcting for the bias toward older articles. However, as Dr. Blount correctly states, there are a number of limitations to citation analysis that can miss the true impact of certain publications in their relevant field.

Citation analysis uses the total number of citations in a publication's history as a surrogate metric for its impact within a particular field of research. With Google Scholar, patents, webpages, and other publications are included along with the peer-reviewed articles. The assumption that must be made for this type of analysis is that the number of times another document has cited a publication is a relative statistic that correlates directly with the influence of the given publication within its field. As Dr. Blount correctly states, however, this may not be an entirely accurate measurement of impact as it fails to take into account the quality of the research presented, the relative influence of the people and institutions it has reached, and any true influence on the direction of future research and policy making. However, it is still thought to be a good measure, at the least, of short-term research impact. ${ }^{6}$

Unfortunately, as anyone attempting to study subjective or non-empirical phenomena can attest, choosing a suitable quantifiable parameter to assign a mathematical value to a qualitative property is challenging. For the determination of a publication's contribution to a given scientific discipline, several different indices have been proposed. ${ }^{2}$ Two of them were mentioned by Dr. Blount: the Hirsch index (or h-index) and the replicability index (or r-index).

The first of these, the h-index, is used to evaluate the relative contributions of an individual author or institution based on their publications and the number of times these publications have been cited. ${ }^{4}$ This is certainly a good measure of the output of an individual. However, as Dr. Blount mentions, it cannot account for certain factors, such as the true contribution of an author (being the first author on a few papers vs making a small contribution to many papers will introduce bias). It is also subject to the same issues many researchers face in their careers-grant awards, tenure decisions, and other opportunities are frequently influenced simply by the number of publications an individual has to their name, possibly favoring authors who have published multiple articles of relatively low importance over those who have published fewer articles with considerably more substance. This practice may also give less importance to researchers who are relatively new in their careers even if their contributions are revolutionary and novel in content. The impact factor of the journals in which articles are published is another indicator of the caliber of a paper, but it also traces back to the shortcomings of the h-index: a journal's impact factor is calculated by the average number of citations to its published articles. Thus, these methods still ultimately rely on citation counts. ${ }^{6}$

In the field of neurosurgery, as with other branches of medicine, articles that may be used for the development of therapeutic practices and diagnostic tests are carefully scrutinized and ranked based on their experimental design and rigor: level I for randomized controlled trials, level II for prospective studies, level III for retrospective studies, etc. For this type of evaluation then, the r-index is possibly the best method for quantifying publications' scientific values. This method utilizes power statistics to estimate-given the sample sizes, variable measurements, and number of significant results in a set of studies-how likely another significant result will be if the study were to be repeated another time. ${ }^{5}$ Indeed, replicability is the hallmark for scientific proof, and the failure to replicate 
findings has brought down major studies that had garnered copious interest and excitement (though it could be argued that such studies still have a weighty impact: after retracting two infamous studies claiming to induce pluripotency in differentiated cells through stress, Nature, after an inability to replicate results, ended up revising its editorial policies). ${ }^{1}$ Though a useful measure for scientific merit, the complexity of calculating an r-index and the need to examine multiple publications limit the index's feasibility as an efficient means of determining impact.

Meta-analyses are considered the highest level of evidence, especially if they analyze all the relevant randomized controlled trials and/or prospective studies. In this sense, publications without original research, other than bibliometric analyses, are deemed to be the most influential, and the inclusion of original studies in such reviews does significantly determine their impact on their given scientific discipline.

So, several different values may give meaning to a publication's impact in their own right. A truer measure of scientific influence would take into account each of these indices - the number of times an article has been cited, the impact factor of the journal it appears in, the overall contribution of its authors, the replicability of the studies, and others. As the number of publications in circulation continues to grow and the computational capabilities of the community expands, highly sophisticated algorithms and even possibly machine learning in the future may give us greater abilities to estimate a publication's true worth.

\section{References}

1. Cyranoski D: Papers on 'stress-induced' stem cells are retracted. High-profile reports claiming an easy way to create pluripotent cells were flawed, Nature announces. Nature News. July 2, 2014 (https://www.nature.com/news/papers-onstress-induced-stem-cells-are-retracted-1.15501) [Accessed August 17, 2018]

2. Ellegaard O, Wallin JA: The bibliometric analysis of scholarly production: how great is the impact? Scientometrics 105:1809-1831, 2015

3. Harzing: About publish or perish, in Publish or Perish 4 User's Manual. St. Albans: Tarma Software Research Ltd, 2016

4. Hirsch JE: An index to quantify an individual's scientific research output. Proc Natl Acad Sci U S A 102:16569, 2005

5. Schimmack U: A revised introduction to the r-index. Replicability-Index: Improving the Replicability of Empirical Research. January 31, 2016 (https:// replicationindex.wordpress.com/2016/01/31/a-revisedintroduction-to-the-r-index/) [Accessed August 17, 2018]

6. Wallin JA: Bibliometric methods: pitfalls and possibilities. Basic Clin Pharmacol Toxicol 97:261-275, 2005

\section{INCLUDE WHEN CITING}

Published online October 19, 2018; DOI: 10.3171/2018.7.PEDS18389. 